\title{
Epigenetic remodeling regulates transcriptional changes between ovarian cancer and benign precursors
}

\author{
Kevin M. Elias, ${ }^{1,2,3}$ Megan M. Emori, ${ }^{1,4}$ Thomas Westerling, ${ }^{5}$ Henry Long, ${ }^{5}$ Anna Budina-Kolomets, ${ }^{6}$ \\ Fugen Li, ${ }^{5}$ Emily MacDuffie, ${ }^{1}$ Michelle R. Davis, ${ }^{1,2,3}$ Alexander Holman, ${ }^{7}$ Brian Lawney, ${ }^{7}$ \\ Matthew L. Freedman, ${ }^{1,5,8}$ John Quackenbush, ${ }^{7}$ Myles Brown, ${ }^{5}$ and Ronny Drapkin ${ }^{1,3,6}$ \\ 'Department of Medical Oncology, Dana-Farber Cancer Institute, Boston, Massachusetts, USA. 'Division of Gynecologic \\ Oncology, Department of Obstetrics and Gynecology and Reproductive Biology, Brigham and Women's Hospital, Boston, \\ Massachusetts, USA. ${ }^{3}$ Harvard Medical School, Boston, Massachusetts, USA. ${ }^{4}$ Graduate School of Arts and Sciences, \\ Harvard University, Cambridge, Massachusetts, USA. ${ }^{5}$ Center for Functional Cancer Epigenetics, Dana-Farber Cancer \\ Institute, Boston, Massachusetts, USA. ${ }^{6}$ Penn Ovarian Cancer Research Center, Department of Obstetrics and Gynecology, \\ Perelman School of Medicine, University of Pennsylvania, Philadelphia, Pennsylvania, USA. ${ }^{7}$ Computational Biology and \\ Functional Cenomics Laboratory, Dana-Farber Cancer Institute, Harvard School of Public Health, Boston, Massachusetts, \\ USA. ${ }^{8}$ Center for Cancer Genome Discovery, Dana-Farber Cancer Institute, Boston, Massachusetts, USA.
}

Regulation of lineage-restricted transcription factors has been shown to influence malignant transformation in several types of cancer. Whether similar mechanisms are involved in ovarian cancer pathogenesis is unknown. PAX8 is a nuclear transcription factor that controls the embryologic development of the Müllerian system, including the fallopian tubes. Recent studies have shown that fallopian tube secretory epithelial cells (FTSECs) give rise to the most common form of ovarian cancer, high-grade serous ovarian carcinomas (HCSOCs). We designed the present study in order to understand whether changes in gene expression between FTSECs and HCSOCs relate to alterations in PAX8 binding to chromatin. Using whole transcriptome shotgun sequencing (RNA-Seq) after PAX8 knockdown and ChIP-Seq, we show that FTSECs and HCSOCs are distinguished by marked reprogramming of the PAX8 cistrome. Cenes that are significantly altered between FTSECs and HCSOCs are enriched near PAX8 binding sites. These sites are also near TEAD binding sites, and these transcriptional changes may be related to PAX8 interactions with the TEAD/YAP1 signaling pathway. These data suggest that transcriptional changes after transformation in ovarian cancer are closely related to epigenetic remodeling in lineage-specific transcription factors.

Conflict of interest: The authors have declared that no conflict of interest exists.

Submitted: April 11, 2016 Accepted: July 12, 2016 Published: August 18, 2016

Reference information: JCI Insight. 2016;1(13):e87988. doi:10.1172/ji.insight.87988.

\section{Introduction}

The genome-wide map of transcription factor binding sites is known as the cistrome (1). Transcription factors which are unique to a particular tissue or cell type are referred to as lineage-defining transcription factors (2). Changes in the binding of lineage-defining transcription factors have been shown to be key transformational events in several malignancies, including melanoma, gastrointestinal stromal tumors, and germ cell tumors (3-7).

PAX8 is a lineage-defining transcription factor that directs the development of the Müllerian duct (the female reproductive tract) and also identifies malignancies derived from Müllerian epithelia (8-10). There is an emerging consensus that the majority of high-grade serous carcinomas, the most common type of ovarian cancer, are derived from PAX8-expressing cells in the distal fallopian tube (11-13). We have previously demonstrated the biologic plausibility of this hypothesis by showing that dysregulation of BRCA1/2 and TP53 in PAX8-expressing mouse or human fallopian tube secretory epithelial cells (FTSECs) can induce tumors that are genomically and phenotypically identical to human high-grade serous ovarian carcinomas (HGSOCs) (14-17). However, whether alterations in the PAX8 cistrome contribute to ovarian cancer pathogenesis remains unknown. Here, we explore the transcriptional network regulated by PAX8 and present evidence for PAX8 reprogramming during HGSOC transformation. 


\section{Results}

PAX8 loss alters the transcriptome of ovarian cancer cells but not benign fallopian tube secretory cells. For this study, we selected cell lines that reflected the genomic alterations observed in The Cancer Genome Atlas (TCGA) (18-21). Three high-grade serous cancer lines, for which we have previously published detailed genomic and phenotypic data (KURAMOCHI, OVSAHO, and JHOS4), were compared with 3 immortalized fallopian tube secretory cell lines (FT33, FT194, and FT246), which were generated in our laboratory $(15,18,22,23)$. We began with a RNA-Seq analysis to study the transcriptional network regulated by PAX8. We knocked down PAX8 in all 6 cell lines using siRNA. For each cell line, we used 2-3 distinct siRNA sequences, plus a pooled siRNA, and compared these with both a nontargeting siRNA and nontransfected cells (18). The efficiency of PAX8 knockdown was assessed both by the number of PAX8 transcript reads, as determined by the sequencing, and Western blot (Supplemental Figure 1, A and B; supplemental material available online with this article; doi:10.1172/jci.insight.87988DS1). We then performed RNA-Seq on all samples, followed by unsupervised clustering of the transcripts most affected by loss of PAX8 expression.

As expected, the specimens clustered distinctly into benign and malignant groups (Figure 1A). Among the benign cell lines, very few transcripts (1 for FT33, 13 for FT194, and 32 for FT246) were significantly altered by PAX8 loss at an adjusted $P$ value $<0.05$, while in contrast, among the cancer cell lines, the effect of PAX8 loss varied from cell line to cell line, ranging from 12 transcripts in JHOS4 to 135 transcripts in KURAMOCHI and 541 transcripts in OVSAHO (Supplemental Dataset 1). The surprisingly few number of transcripts impacted by PAX8 knockdown is in part a result of the stringent statistical threshold we selected to account for multiple testing, but even when using the unadjusted $\alpha$ values for the transcriptional changes, the cancer cell lines were much more affected by PAX8 loss than the benign cell lines (Supplemental Dataset 1). We considered that the relative lack of transcriptional impact from PAX8 loss in the fallopian tube cell lines might be due to functional redundancy between PAX8 and other PAX family homologs. However, this appeared unlikely, as the RNA-Seq data revealed that there were no other PAX homologs that were substantially expressed among the cell lines (Figure 1B).

We also considered the possibility that PAX8 knockdown would induce apoptosis and that the transcriptome signature changes would simply reflect cell death. To determine this, we performed proliferation assays after PAX8 knockdown on 3 of the lines used in this study, KURAMOCHI, OVSAHO, and FT194. As positive controls, we used 2 cell lines we have previously reported as being dependent on PAX8 for cell growth, IGROV-1 and OVCAR3 (24). As a negative control, we used MCAS, a mucinous carcinoma cell line that does not express PAX8. While PAX8 knockdown did reduce cell proliferation in KURAMOCHI, the cell lines OVSAHO and FT194 were unaffected by PAX8 knockdown (Supplemental Figure 1C), suggesting that transcriptional changes were not a proxy for cell death.

To investigate how the transcriptomes differed between benign and malignant cells more globally, we used the control samples from the RNA-Seq experiment (i.e., both the untransfected and nontargeting siRNA controls, ignoring the PAX8 knockdown experiments) to look for the most differentially regulated transcripts, irrespective of PAX8 dependence. At a threshold of a $\log _{2}$-fold change in expression and $P<0.05$, there were 1,343 downregulated genes and 951 upregulated genes that distinguished FTSECs from HGSOCs (Supplemental Dataset 2). A gene ontology analysis of these genes showed enrichment for genes regulating cell proliferation, blood vessel development, and cell adhesion (Table 1) — processes that are important in development and malignancy but likely less important for the maintenance of a mature epithelium.

Ovarian cancer cells show marked reprogramming of the PAX8 cistrome. We hypothesized that the lack of PAX8 transcriptional dependence in the FTSEC lines could be due to epigenetic remodeling around PAX8 binding sites. Therefore, we tested whether PAX8 binding was different in FTSECs compared with HGSOCs by mapping the PAX8 cistrome in all 6 cell lines using ChIP-Seq (Figure 2A and Supplemental Table 1) (25). Peaks among the cell lines were aligned to define a minimum consensus binding motif for PAX8 for FTSECs and HGSOC (Figure 2B). The $12 \mathrm{bp}$ motifs were identical, except for slight variation at the second, sixth, seventh, and eighth bases. As the consensus binding motif for PAX8 has not been previously defined, the closest known motif to our identified PAX8 motif in all 6 cell lines was the PAX2 motif, validating that these are in fact PAX family homologs.

Among the 6 cell lines, there were 11,361 unique PAX8 peaks identified at a false discovery rate of $<0.01$, with a median of 2,886 peaks per sample (range 1,122-5,984) (Supplemental Dataset 3). Unsupervised clustering of the PAX8 cistromes separated the specimens distinctly into benign and malignant groups (Figure 2C). This appeared to be driven by the high homology among the FTSEC cistromes and 


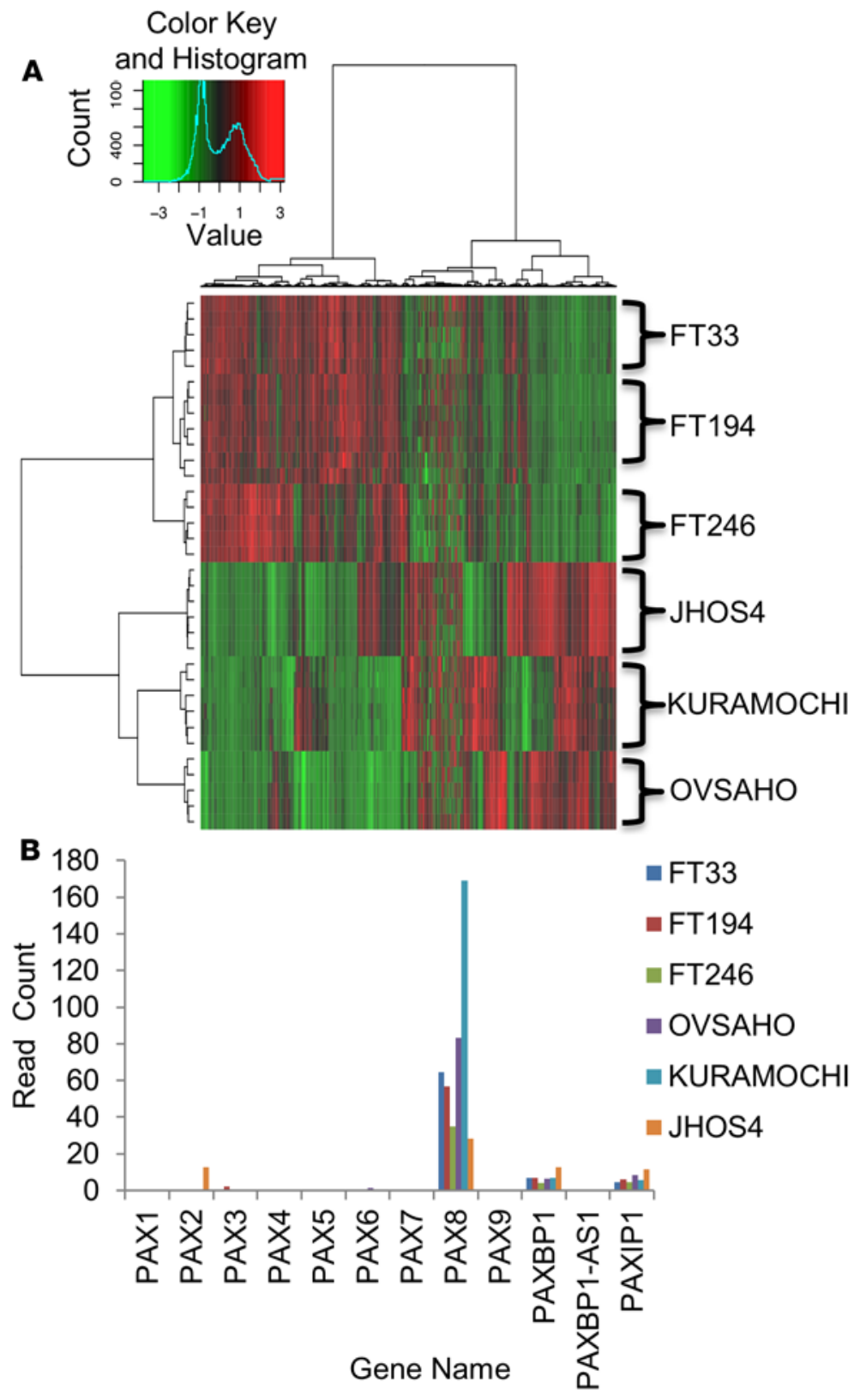

Figure 1. Impact of PAX8 knockdown on cell line transcriptomes. Benign fallopian tube secretory epithelial cells (FTSEC, designated FT33, FT194, and FT246) and high-grade serous ovarian cancer cell lines (HGSOC, known as OVSAHO, KURAMOCHI, and JHOS4) were subjected to PAX8 siRNA knockdown followed by RNA-Seq. (A) $\mathrm{K}$-means clustering of top 100 most differentially expressed transcripts for each cell line. There are 5-6 transcriptome results for each cell line (untransfected control, nontargeting control, and 3-4 siRNA replicates). (B) From the same dataset, expression of the PAX homologs in each cell line.

marked diversity among the HGSOC cistromes. Although cancer cell lines had more PAX8 binding sites overall, the relative frequency of PAX8 binding sites among DNA coding and noncoding regions was consistent across benign and malignant cell lines (Figure 3). Notably, only a small percentage of peaks $(726 / 11,361 ; 6.4 \%$; 95\% CI, $6.0 \%-6.9 \%)$ overall were near promoter regions. The vast majority of peaks $(9,912 / 11,361 ; 87.2 \% ; 95 \%$ CI, 86.6\%-87.9\%) were located in either intronic or intergenic regions. Further analysis identified 3 sets of PAX8 peaks: i) a highly conserved set among all 6 cell lines composed of 558 peaks; ii) a set of peaks common to benign cell lines but absent in cancer cell lines composed of 3,013 peaks; and iii) a very heterogeneous pattern of peaks unique to each cancer cell line, ranging from a few unique PAX8 peaks (including JHOS4) to more than 3,000 unique PAX8 peaks (including both KURAMOCHI and OVSAHO) (Figure 4).

Differentially expressed genes between ovarian cancer cells and benign fallopian tube cells are clustered near PAX8 binding sites. The ChIP-Seq data confirmed our hypothesis that the PAX8 cistrome differs in benign and malignant contexts, so we returned to the RNA-Seq data to determine how this might contribute to differences in the transcriptomes between benign and malignant cells. Among the 2,294 genes that distinguished FTSECs from HGSOCs, there were 415 downregulated genes and 171 upregulated genes 
Table 1. Gene ontology analysis of PAX8 target genes differentially expressed between malignant and benign cell lines.

\begin{tabular}{llcc}
\hline Gene ontology pathway & P value & FDR & Hits in pathway \\
Regulation of cell proliferation & $3.5 \times 10^{-12}$ & $9.3 \times 10^{-9}$ & 66 \\
Blood vessel development & $1.0 \times 10^{-10}$ & $1.4 \times 10^{-7}$ & 32 \\
Vasculature development & $1.9 \times 10^{-10}$ & $1.7 \times 10^{-7}$ & 32 \\
Cell adhesion & $4.5 \times 10^{-10}$ & $3.0 \times 10^{-7}$ & 57 \\
Biological adhesion & $4.6 \times 10^{-10}$ & $2.5 \times 10^{-7}$ & 57 \\
Blood vessel morphogenesis & $2.6 \times 10^{-8}$ & $1.2 \times 10^{-5}$ & 26 \\
Positive regulation of cell proliferation & $2.9 \times 10^{-8}$ & $1.1 \times 10^{-5}$ & 38
\end{tabular}

within 50,000 bp of a PAX8 binding site (Table 2; Supplemental Dataset 4). Therefore, although only $6.4 \%$ of PAX8 binding sites were near a gene promoter region, $586 / 726$ of these peaks $(80.7 \%$; $95 \%$ CI, $77.7 \%-83.4 \%$ ) were near a gene with significantly different expression between normal and malignant cells. This is compared with 1,708 genes with altered expression that were distant from one of the other 11,221 PAX8 peaks $(13.2 \%$; 95\% CI, 12.6\%-13.8\%) for an odds ratio of an altered gene being near a PAX8 binding site of 27.5 (95\% CI, 22.7\%-33.3\%; $P<10^{-7}$; Fisher's exact test).

Alterations in the PAX8 cistrome in cancer lines are associated with increased interactions between PAX8 and TEAD. In other malignances, changes in the function of lineage-defining transcription factors are influenced by interaction with transcriptional coregulators, so we questioned whether the differences in expression of genes near PAX8 binding sites could be the result of altered interactions between PAX8 and a second transcription factor. We reanalyzed our ChIP-Seq data for other consensus binding motifs that overlapped the PAX8 peaks. Looking at the total PAX8 cistromes of all 6 cell lines, the second or third most common binding motif after PAX8 in each cell line was the TEAD family consensus binding motif (Supplemental Table 2 and Figure 5A). We found that all 4 homologs in the TEAD family were expressed in all the cell lines by RNA-Seq and confirmed TEAD family expression by immunofluorescence (Supplemental Figure 2, A and B). Of note, loss of PAX8 did not affect expression of any of the TEAD factors themselves (Supplemental Dataset 1). TEAD family members are key members of the Hippo/ Yes-associated protein (YAP) signaling pathway, an evolutionarily conserved pathway that controls organ size, cell proliferation, and apoptosis. Importantly, YAP1, the major downstream effector of the Hippo pathway, was also expressed in all the cell lines (Supplemental Dataset 1 and Supplemental Figure 2B).

Therefore, we hypothesized that PAX8, YAP1, and TEAD factors might be interacting. YAP does not have a DNA binding domain but is rather a transcriptional cofactor. TEAD family members do have DNA binding domains, however, so we examined the genes in Table 2 for overlap between PAX8 peaks and TEAD motifs (Supplemental Dataset 5). As shown in Table 2, TEAD motifs within PAX8 peaks were overrepresented among genes showing higher expression in cancer lines than among those showing lower expression among cancer lines (13/30 genes versus $4 / 30$ genes, $P=0.02$, Fisher's exact test). These results raised the possibility that PAX8 and TEAD factors may physically and functionally interact.

To address whether PAX8 physically interacts with TEAD, we used a proximity ligation assay (PLA) and coimmunoprecipitation assay. With PLA, the use of 2 antibodies against different proteins allows both localization and quantification of protein-protein interactions. The unique amplification of signal provided by the detection steps enables one to study both stable and transient interactions at endogenous expression levels of the target protein(s). The known interaction between PAX8 and the histone acetyltransferase ep300 was used as a positive control (26). Using PLA, we found that PAX8, pan-TEAD, and YAP1 physically interact in Müllerian cells (Figure 5B and Supplemental Figure 3). The interactions were concentrated in the nucleus, as expected for transcription factors. The specificity of this interaction was demonstrated by knocking down PAX8 with siRNA (Supplemental Figure 3), which resulted in the loss of the PLA interaction signal. We confirmed the formation of a PAX8-TEAD complex using a coimmunoprecipitation assay to show that TEAD is detected in the precipitate after PAX8 pull-down (Figure 6). 


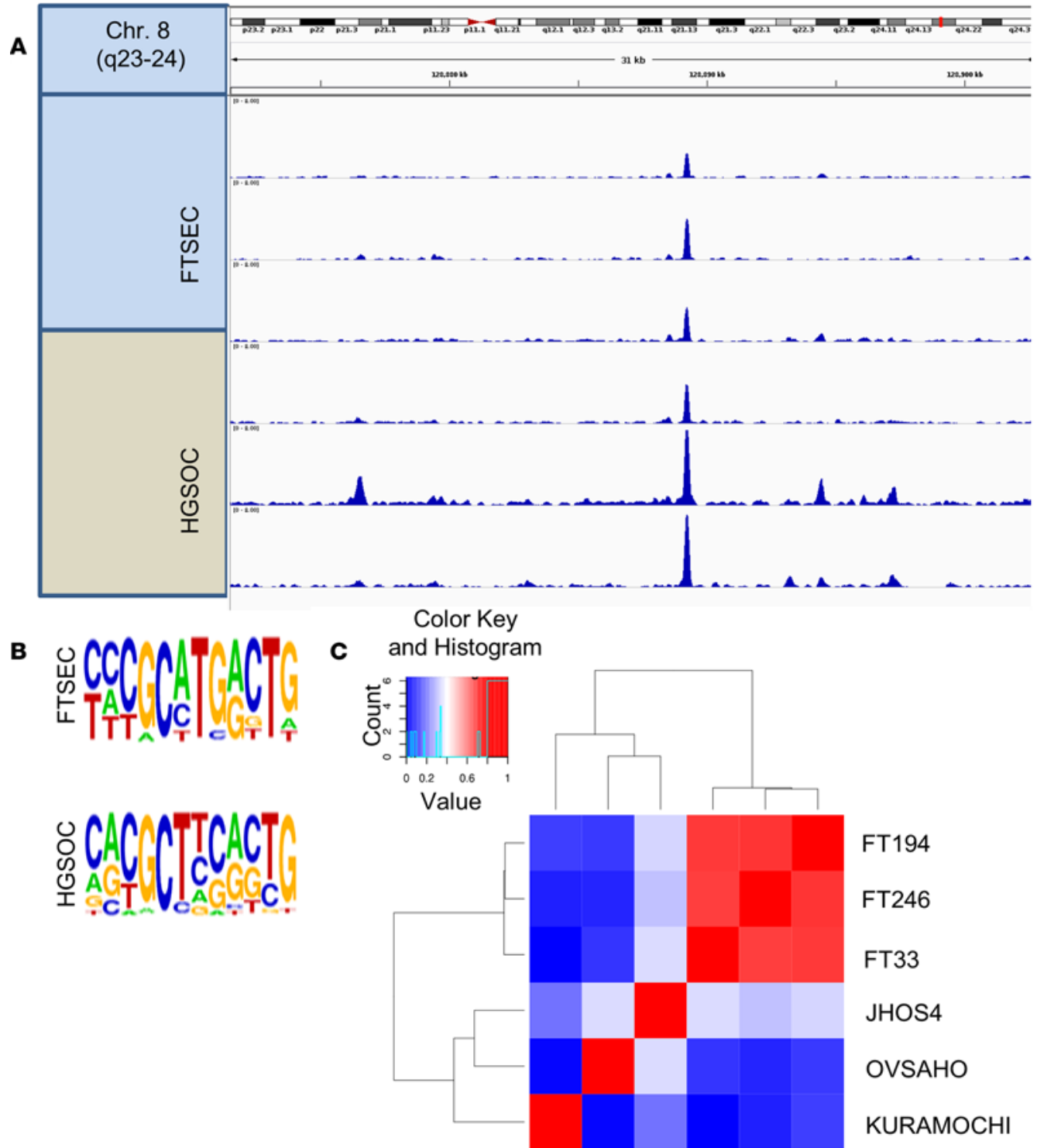

Figure 2. Genome-wide PAX8 binding in FTSEC and HGSOC. (A) Browser view of highly conserved ChIP-Seq peak on 8q23-24. (B) Consensus binding motif for PAX8 in FTSEC and HCSOC lines. (C) Unsupervised pairwise correlation of the PAX8 cistromes from all cell lines.

\section{Discussion}

The PAX genes are a family of homologs of the Drosophila gene paired. Following the original report describing its cloning in 1986, it soon became apparent that paired was the prototype of a larger network of regulatory genes responsible for segmentation, patterning, and organ development $(27,28)$. Later, these genes were organized into the PAX family owing to their characteristic 128-amino acid N-terminal DNA-binding domain. PAX homologs are highly conserved through evolution, appearing in the genomes of nematodes through humans (29). They are nuclear transcription factors, displaying both sequence-specific recognition and transcriptional regulation $(30,31)$. During embryogenesis, PAX8 is expressed predominantly in the midbrain-hindbrain boundary, kidney, thymus, thyroid, and Müllerian system but, in the adult expression, is limited to the thyroid, Müllerian system, and cells in the convoluted tubules of the renal nephron $(8,32)$. $\mathrm{Pax}^{-1-}$ female mice display infertility, owing to a functional defect in the development of the Müllerian tract (10). The fact that PAX8 expression is retained in $99 \%$ of high-grade serous cancers has made it a transcription factor of considerable interest for understanding the pathogenesis of this disease (9). Interestingly, $P A X 8$ is the single most differentially expressed gene when comparing ovarian cancer cell lines with all other cancer cell lines in the Cancer Cell Line Encyclopedia (24). Loss of $P A X 8$ in most, but not all, serous 
Table 2. Top differentially expressed genes between malignant and benign cell lines located near a PAX8 binding site.

\begin{tabular}{|c|c|c|c|c|c|}
\hline \multicolumn{6}{|c|}{ Upregulated PAX8 target genes } \\
\hline Gene symbol & Log fold change & $\begin{array}{l}\text { TEAD motif within } \\
\text { PAX8 peak }\end{array}$ & Gene symbol & Log fold change & $\begin{array}{l}\text { TEAD motif within } \\
\text { PAX8 peak }\end{array}$ \\
\hline S100A1 & 7.5 & NO & KLK8 & 5.2 & NO \\
\hline BMP7 & 6.9 & YES & CLDN4 & 4.8 & NO \\
\hline S100A4 & 6.8 & NO & EMX20S & 4.8 & YES \\
\hline WFDC2 & 6.4 & NO & ID4 & 4.8 & YES \\
\hline SPON1 & 6.3 & YES & LEMD1 & 4.7 & NO \\
\hline KLK6 & 6.0 & NO & LGR5 & 4.7 & YES \\
\hline COL26A1 & 5.8 & YES & GLDC & 4.6 & YES \\
\hline APOA1 & 5.8 & YES & РTP4A3 & 4.6 & NO \\
\hline PTCS1 & 5.7 & NO & ALPL & 4.6 & NO \\
\hline EMX2 & 5.7 & NO & TNNC1 & 4.5 & NO \\
\hline SEPP1 & 5.7 & NO & $\mathrm{BOC}$ & 4.5 & YES \\
\hline TMEM100 & 5.5 & YES & CRABP1 & 4.4 & NO \\
\hline GPX3 & 5.4 & NO & $\mathrm{PI3}$ & 4.2 & NO \\
\hline SLC34A2 & 5.3 & YES & TMPRSS3 & 4.2 & NO \\
\hline CDH6 & 5.3 & YES & FAM107A & 4.2 & YES \\
\hline \multicolumn{6}{|c|}{ DownregulatedPAX8 target genes } \\
\hline Gene symbol & Log fold change & $\begin{array}{l}\text { TEAD motif within } \\
\text { PAX8 peak }\end{array}$ & Gene symbol & Log fold change & $\begin{array}{l}\text { TEAD motif within } \\
\text { PAX8 peak }\end{array}$ \\
\hline SERPINE1 & -9.0 & NO & EDIL3 & -5.8 & NO \\
\hline FN1 & -8.7 & YES & $A X L$ & -5.8 & NO \\
\hline COL1A1 & -8.6 & NO & CDKN1A & -5.6 & NO \\
\hline MT1E & -8.6 & NO & CD70 & -5.6 & NO \\
\hline SAA1 & -8.6 & NO & RAC2 & -5.4 & NO \\
\hline SAA2 & -7.4 & NO & GLIPR1 & -5.3 & NO \\
\hline SLC16A3 & -7.0 & NO & SLC38A5 & -5.3 & NO \\
\hline MT1L & -6.6 & NO & THBS1 & -5.3 & NO \\
\hline SRGN & -6.5 & YES & RRAD & -5.3 & NO \\
\hline TGFBI & -6.3 & NO & CAPG & -5.2 & NO \\
\hline CYGB & -6.2 & NO & CXCL1 & -5.2 & NO \\
\hline COL8A1 & -5.9 & NO & CXCL5 & -5.1 & NO \\
\hline GREM1 & -5.9 & NO & CRISPLD2 & -5.1 & YES \\
\hline MIR492 & -5.9 & YES & COL6A1 & -5.1 & NO \\
\hline COL5A2 & -5.9 & NO & C1QTNF1 & -5.1 & NO \\
\hline
\end{tabular}

ovarian cancer cell lines leads to apoptosis and decreased tumorigenicity, while cancer cell lines from other organ sites are unaffected $(24,33)$. Together, these findings have led to substantial interest in $P A X 8$ as a lineage-defining gene for Müllerian cancers in humans.

While other studies on the PAX8 cistrome have focused on its role in thyroid cells, ours is the first detailed analysis to our knowledge of the PAX8 cistrome in Müllerian epithelia (34). Here, we have analyzed the cistrome of PAX8 to elucidate its interactions with chromatin in both benign and malignant Müllerian epithelia and found that the function of PAX8 appears to be context dependent. There is a shared set of PAX8 binding sites among benign and malignant Müllerian epithelia, but with transformation, PAX8 appears to lose a stereotypical set of binding sites while, at the same time, gaining access to an array of tumor-specific binding sites. As the 3 FTSEC lines used in this study were derived from 3 different patients and generated using 3 different strategies for immortalization, the high degree of overlap in PAX8 binding sites among the FTSEC lines likely represents a highly conserved cistrome and not simply stochastic changes due to individual variation or experimental artifact. In contrast, the variation seen among the HGSOC cell lines may be driven by underlying differences in the biology of individual 


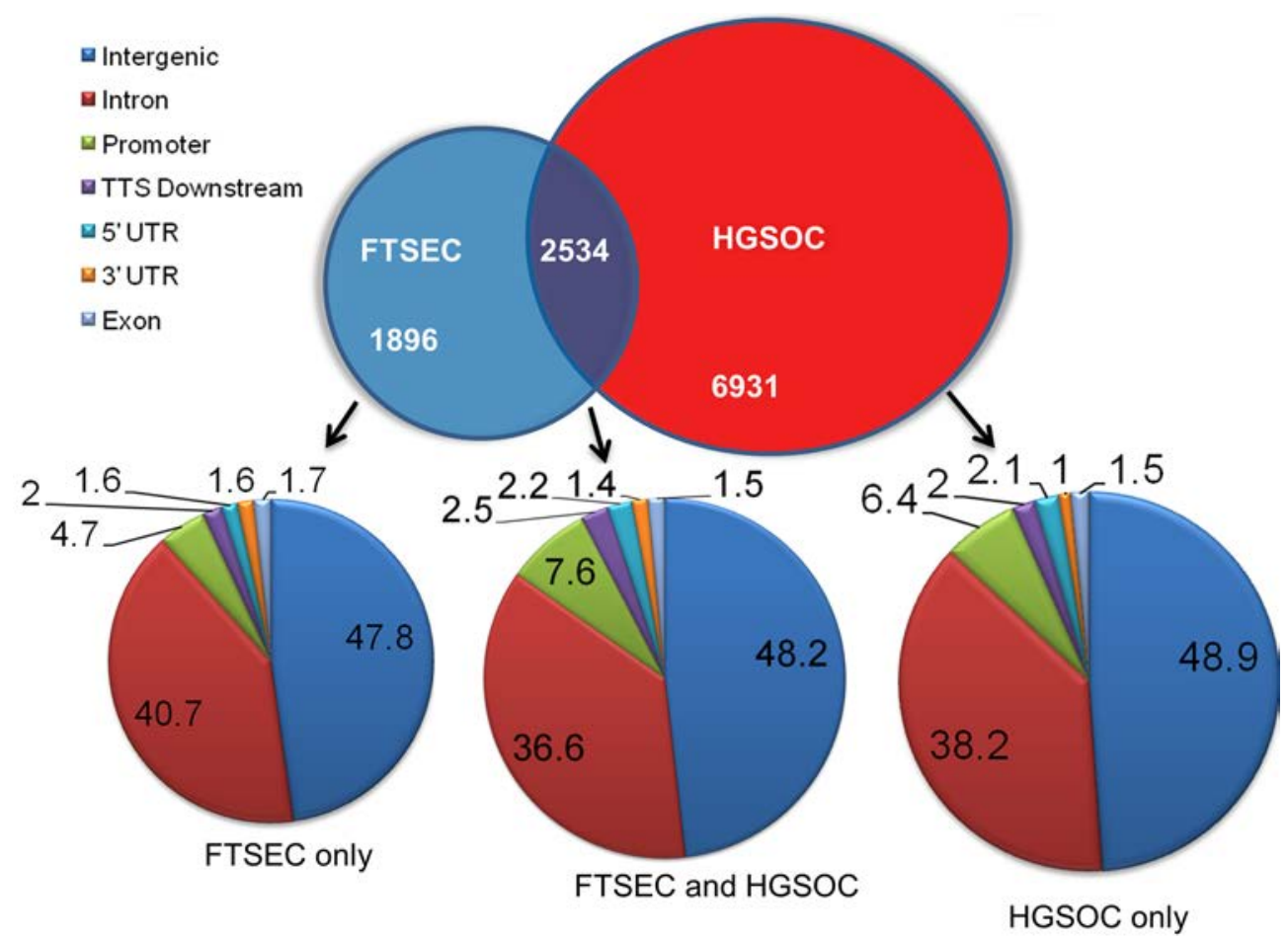

Figure 3. Unique PAX8 binding sites by cell line type and genomic locations of binding sites. The relative frequency of PAX8 binding sites among genomic elements was similar among the benign and malignant cell lines.

tumors, including copy number variation and mutations. Even so, among the cancer cell lines, there were shared PAX8 binding sites not seen among the FTSEC lines near several genes that have previously been associated with HGSOC, including LGR5, ID4, PI3 (which encodes the protein Elafin), and WFDC2 (which encodes the protein HE4) (35-42).

Surprisingly, loss of PAX8 does not significantly alter gene expression in FTSECs or even all cancer cell lines. Our data suggest several possible reasons for this. First, the cell lines most affected by PAX8 loss also have the highest expression of PAX8; indeed, in previous work, we have shown that dependence on PAX8 for cell survival can be related to PAX8 amplification (24). This may explain why the JHOS4 line, which expresses relatively less PAX8 than KURAMOCHI or OVSAHO, had fewer PAX8 peaks identified and was less affected by PAX8 knockdown. Even so, PAX8 is not amplified in any of the cell lines assessed in the current work, and PAX8 can be highly expressed in both benign and malignant cells (18). Another possible explanation for PAX8 behavior is that the majority of PAX8 binding sites are located in intergenic regions. PAX8 may not carry out most of its functions through direct gene regulation, but instead through epigenomic mechanisms; indeed, the conserved PAX8 ChIPSeq peak shown in Figure 1A is near a recently described super-enhancer site (43). In addition, our gene ontology analysis of the RNA-Seq comparison between the FTSEC and HGSOC cell lines showed that most of the genes that distinguish benign and malignant cells are related to morphogenesis and angiogenesis. This could explain why PAX8 is important in embryonic development and cancer but not in a mature epithelium, where these processes are likely epigenetically repressed. Finally, our motif and RNA-Seq analyses suggest a possible mechanism for this regulation consisting of interactions between PAX8 and TEAD signaling. TEAD homologs are transcriptional cofactors, essential for the Hippo/YAP signaling pathway. Recently, we showed that TEAD/YAP signaling plays a key role in the transformation of fallopian tube secretory cells into high-grade serous carcinomas (22). YAP1 lacks an intrinsic DNA-binding domain and requires interaction with transcription factors to mediate its oncogenic function. PAX proteins have been shown to be contextual transcriptional regulators of YAP signaling (44). For example, an interaction between PAX5 and TEAD4 has been recently described (45). Our current data suggest that PAX8 may function similarly in the context of the Müllerian epithelium. Whereas the PAX8-TEAD and PAX8-ep300 interactions were similar in HGSOC and FTSEC cell lines, only the cancer cell lines demonstrated a high number of interactions between PAX8 and YAP1, suggesting a possible mechanism for altered PAX8 function. Perhaps the subtle differences observed between the PAX8 consensus binding motif in the FTSECs and HGSOCs affect how PAX8, TEAD, and YAP interact on chromatin. Although further experiments will be needed 


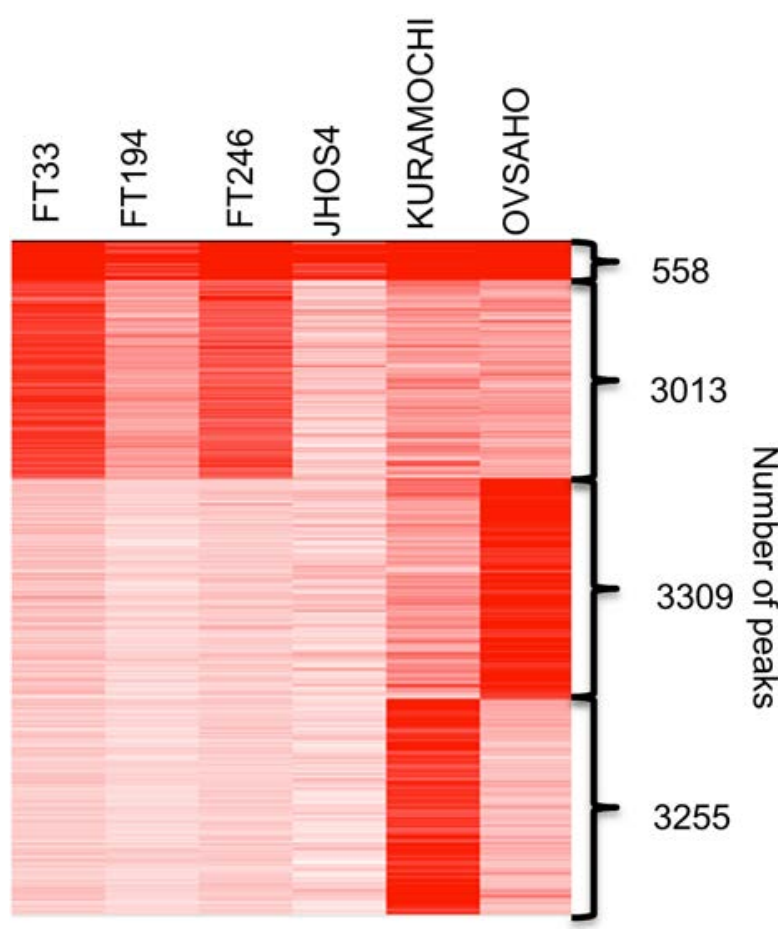

Figure 4. K-means clustering of ChIP-Seq peaks by cell line and number of peaks in common, including FTSEC-specific, and HGSOC-specific peak clusters. Each row represents one peak. to elucidate this connection more fully, similar mechanisms in other malignancies suggest it is plausible that alterations in PAX8 binding may modulate the interactions between YAP/TEAD cofactors at enhancer sites and that PAX8 may be a key mediator of YAP-mediated transformation $(46,47)$.

In conclusion, this study offers some of the first insight to our knowledge into the reorganization of the cistrome during the malignant transformation of Müllerian epithelia. As in other malignances, ovarian cancer pathogenesis involves a dynamic reorganization of chromatin around PAX8, a lineage-specific transcription factor. Understanding how these lineage-specific transcription factors can acquire altered functions in different contexts may reveal new aspects of the pathogenesis of ovarian cancer and other malignancies.

\section{Methods}

Cell culture. KURAMOCHI, OVSAHO, IGROV-1, and JHOS4 cells were a gift from Gottfried Konecny (UCLA, Los Angeles, California, USA). OVCAR3 was purchased from American Type Culture Collection. These cancer cell lines were cultured in DMEM:F12 media (Mediatech Inc.) supplemented with 10\% FBS (Atlanta Biologicals) and 1\% penicillin/ streptomycin (Gibco, Invitrogen). FT33, FT194, and FT246 cells were derived from human fallopian tube secretory cells as previously described and were cultured in DMEM:F12 supplemented with 2\% Ultraser G (Pall Corporation) and 1\% penicillin/streptomycin (23). The 3 FTSEC cell lines were derived from primary FT cells from 3 different patients. FT33 was immortalized by stable expressing human telomerase reverse transcriptase (hTERT) and using 2 separate vectors to stable express SV40 large T antigen and small t antigen. FT194 was immortalized by stable expressing hTERT and using a single vector containing SV40 large $\mathrm{T}$ and small $\mathrm{t}$ antigen. FT246 cells were immortalized by stable expression of hTERT, $p 53$ shRNA, and $\mathrm{CDK} 4^{\mathrm{R} 24 \mathrm{C}}$. All cells were incubated at $37^{\circ} \mathrm{C}$ with $5 \% \mathrm{CO}_{2}$. The identities of the cancer cell lines JHOS4, OVSAHO, and KURAMOCHI were authenticated by typing 10 short tandem repeat (STR) loci using the PowerPlex 2.1 System (Promega). All the FT lines developed in our lab have also been STR fingerprinted. Normal samples for STR analysis were obtained from 10 unmatched lymphoblast cultures (Coriell Institute for Medical Research).

ChIP-Seq. ChIP was performed as described (1). Briefly, cells were grown to confluency on $3 \times 15 \mathrm{~cm}$ to $4 \times 15 \mathrm{~cm}$ plates. The cells were cross-linked with $1 \%$ formaldehyde for 10 minutes at room temperature, and the crosslinking reaction was quenched with a solution of $0.125 \mathrm{M}$ glycine and $5 \mathrm{mg} / \mathrm{ml} \mathrm{BSA}$ (IgG free, protease free) in ice-cold PBS. Nuclear pellets were created by serial washes in high-salt buffers, then lysed in 1\% SDS lysis buffer, $\mathrm{pH} 8.0$, and sonicated to an average size of $250 \mathrm{bp}$. The fragmented chromatin was immunoprecipitated overnight by using primary rabbit polyclonal antibody against PAX8 (Novus, NBP1-32440). DNA was purified using Qiaquick PCR purification columns (Qiagen). DNA was quantified using the Qubit dsDNA HS Assay and Qubit 2.0 Fluorometer (Invitrogen), and shearing efficiency was assessed by running an aliquot of each sample on an E-gel agarose gel (Invitrogen). For ChIPSeq, after repairing the DNA ends, sequencing adaptors (Illumina) were ligated to the purified chromatin, and the DNA was amplified for sequencing as described. Sequencing was performed on a HiSeq 2000 platform (Illumina). After purification of the DNA, libraries were generated and sequencing reads were aligned to the human genome (hg19) using Bowtie software. Peaks were called using MACS v2.0.10 with default cutoffs $(\mathrm{q}<0.01)(48)$. Motif enrichment analysis was done using the HOMER package (http://homer.salk.edu//homer/ngs/peaks.html). Primary sequencing data have been deposited in the Gene Expression Omnibus (GEO) database, www.ncbi.nlm.nih.gov/geo, accession number GSE79893.

RNA-Seq. Reverse transfection with RNAiMAX Lipofectamine (Invitrogen) using 3 different predesigned siRNA oligonulceotides (catalogs s15403, s15404, and s15405; Ambion, Invitrogen), a pool of all 3 siRNA oligonucleotides targeting PAX8, a control siRNA oligonucleotide targeting no known mammalian genes, or a no-siRNA control was performed for each cell line, as previously described (18). Samples were plated in duplicated 6-well plates, with one plate assigned for RNA-Seq and the other for Western blot confirmation. 
A

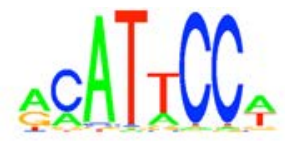

B
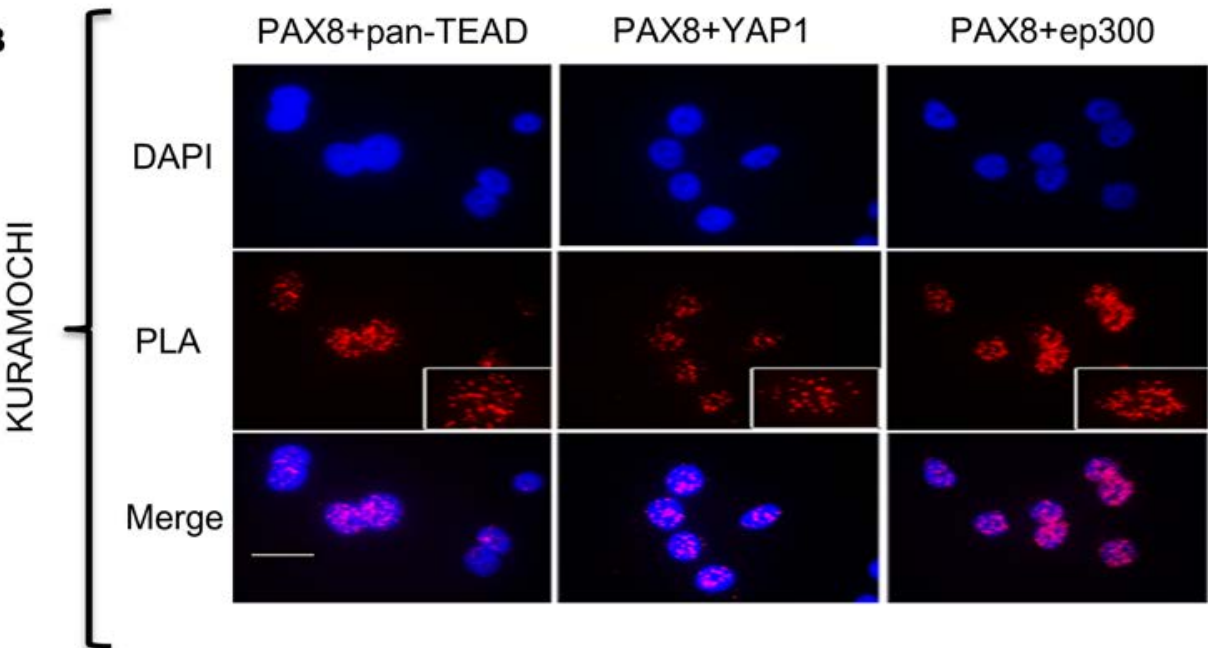

PAX8+pan-TEAD

\section{PAX8+YAP1}

PAX8+ep300
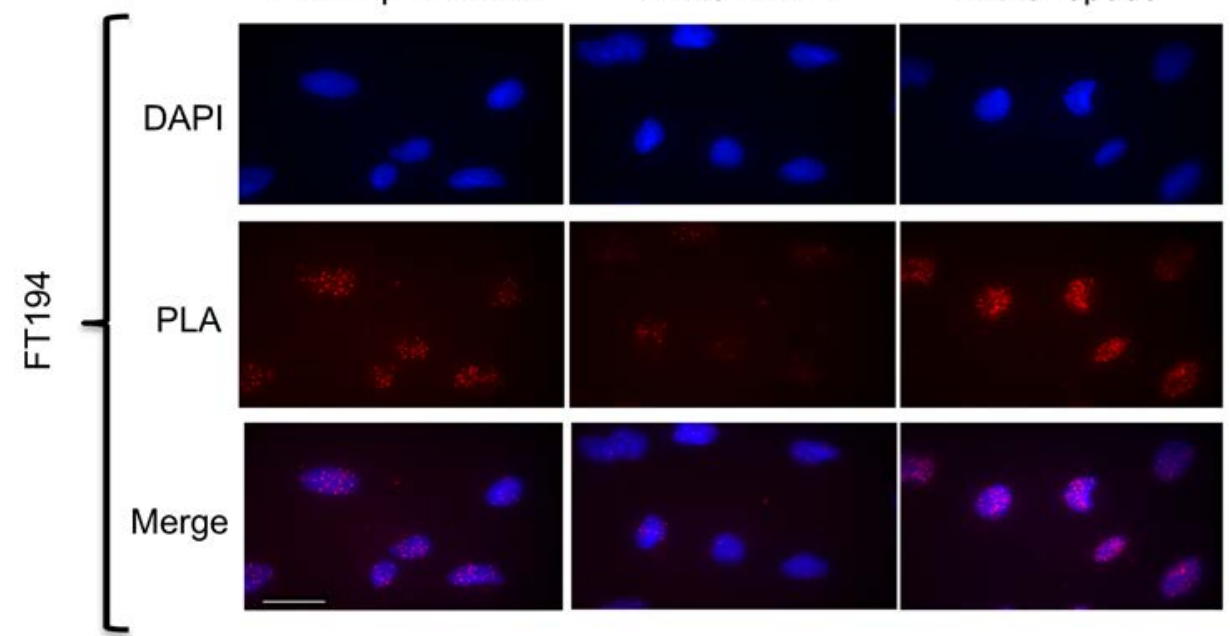

Figure 5. Interaction between PAX8 and TEAD/YAP1. (A) Most common secondary motif found near PAX8 binding sites corresponding to the TEAD motif. See also Supplemental Table 2 and Supplemental Figure 2. (B) An in situ proximity ligation assay (PLA) performed in KURAMOCHI cells showing the interaction between endogenous PAX8 and YAP1 protein and PAX8 and pan-TEAD protein. Each red dot represents a single interaction. Nuclei were counterstained blue with DAPI. Scale bar: $20 \mu \mathrm{m}$. Image magnification $\times 100$. Inset shows $\times 400$ magnification. The previously reported interaction between PAX8 and ep300 serves as a positive control. Cells with efficient PAX8 siRNA knockdown were used as a negative control. See also Supplemental Figure 3. Figures shown are representative of 3 independent experiments.

All cells were incubated for 72 hours before cells were harvested for protein or RNA. For extraction, cells were lysed using RLT buffer. Then, the lysate was homogenized using Qiashredder spin columns (Qiagen). RNA was isolated using RNeasy mini columns (Qiagen) and quantitated using a Nanodrop (Thermo Fisher Scientific), followed by Turbo DNAse treatment (Thermo Fisher Scientific) at $37^{\circ} \mathrm{C}$ for 30 minutes and requantification. After repairing the RNA ends, sequencing adaptors (Illumina) were ligated to the purified RNA, and the RNA was amplified for sequencing. Sequencing was performed on a HiSeq 2000 platform (Illumina). Primary sequencing data have been deposited in the GEO database, accession number GSE83101.

Cell proliferation assays. One thousand, five hundred cells per line were seeded on 96-well plates in quintiplicate to sextuplicate, and siRNA to PAX8 or scramble siRNA was added as described previously (18). After 144 hours, cell density was measured by CellTiter-Glo (Promega). Proliferation was measured relative to the scramble control. 


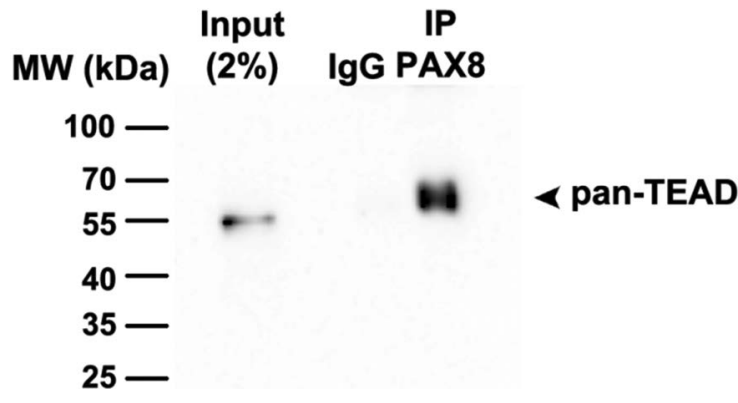

Figure 6. Confirmation of a PAX8-TEAD interaction. Immunoprecipitation of KURAMOCHI nuclear extracts with PAX8 antibody or equal amount of control IgG, followed by Western blot analysis for the level of associated TEAD. Whole nuclear extract from the cells serves as the positive control. Figure shown is representative of 3 independent experiments.
Western blot. Whole cell extracts were created by lysing cell pellets with RIPA buffer (Boston BioProducts) for 20 minutes on ice, and then cleared lysates were quantified by Bradford assay. Samples were loaded onto NuPAGE 4-12\% Bis-Tris gradient gels (Novex, Invitrogen) and separated by electrophoresis in MOPS-SDS running buffer. Proteins were transferred to nitrocellulose membranes via the iBlot dry transfer system (Invitrogen). Blots were blocked for 1 hour at room temperature in 5\% nonfat milk in PBS-Tween-20 (Westnet Inc.) and incubated with a rabbit polyclonal antibody against PAX8 (Proteintech, 10336-1-AP) diluted 1:1,000 in blocking buffer overnight at $4^{\circ} \mathrm{C}$. Blots were then incubated in HRP-linked secondary antibody (GE Healthcare) at 1:4,000 dilution in blocking buffer. Proteins were detected using the ECL2 Western blotting substrate kit (Thermo Fisher Scientific) and imaged with a FluorChem HD2 imager (Cell Biosciences). After initial development, membranes were reprobed with a mouse monoclonal antibody to $\beta$-actin (Sigma-Aldrich, A2228), diluted 1:2,500 in blocking buffer, as a loading control.

Immunofluorescence. For immunofluorescence analysis of endogenous proteins, cells were grown overnight on coverslips in a 6-well culture plate. Cells were then fixed in 4\% paraformaldehyde for 10 minutes, permeabilized in 0.5\% Triton X-100 (Boston BioProducts) for 5 minutes, and incubated with primary and then secondary antibodies at $37^{\circ} \mathrm{C}$ for 1 hour. The following primary antibodies were used: PAX8 (Proteintech, 10336-1-AP), pan-TEAD (Cell Signaling Technology, 13295), YAP (Cell Signaling Technology, 4912), and ep300 (EMD Millipore, NA46). Detection was performed using secondary antibodies conjugated to Alexa Fluor Dyes (Molecular Probes). Cells were then stained with DAPI $(0.5 \mu \mathrm{g} / \mathrm{ml}$, Sigma-Aldrich, D9564) prior to microscopy using a Nikon E400 microscope under $\times 100$ magnification.

PLA. PLA was performed using ovarian cancer cells grown on Lab-Tek II 8-well chamber slides and fixed for endogenous protein detection as described above. The assay was performed using the Duolink kit (Sigma-Aldrich) according to the manufacturer's protocol. Background control was produced by performing parallel experiments in which the 2 primary antibodies were left out of the procedure. A negative control with a cell line that does not express one of the targets was also included. Brightness and contrast were adjusted to allow for the clearest resolution of the images.

Coimmunoprecipitation. The nuclear fraction was isolated from KURAMOCHI cells (Cell Signaling Technology, Cell Fractionation Kit, 9038). Lysate (500 $\mu \mathrm{g}$ ) was immunoprecipitated with $10 \mu \mathrm{g}$ of a rabbit polyclonal antibody against PAX8 (Novus Biologicals, NBP1-32440) or equal amount of rabbit IgG using the Pierce Direct IP kit (Thermo Fisher Scientific, 26148) according to manufacturer's protocol, followed by SDS-PAGE, transfer, and Western blot analysis using a rabbit monoclonal antibody to panTEAD (Cell Signaling Technology, 13295).

Study approval. The current study was exempted from IRB approval, as no study subjects or animals were employed for the described experiments.

Statistics. For ChIP-Seq, sequenced DNA tags were aligned with Bowtie against the reference genome Hg19. Peaks were called using MACS v2.0.10 (https://github.com/taoliu/MACS) with default cutoffs (q $<0.01$ ), and read depth normalized big wiggle files were created. To compare HGSOC and FTSEC cell lines, reads were extracted using High Throughput sequencing, and differential regions were identified by the Differential Expression sequencing package (49). For the comparisons, we considered the union of all peaks from each sample type (HGSOC vs. FTSEC). In Figure 2C, the samples were arranged by unsupervised hierarchical clustering in $\mathrm{R}$ using the Spearman correlations of the top $25 \%$ most-variable peaks. In Figure 4 , the features on the $y$ axis were quantile-normalized and clustered by k-means in R.

For RNA-Seq, sequence tags were mapped to the reference genome Hg19 using TopHat v2.0.6, and transcript levels were calculated as fragments per kb per million mapped reads (FPKM) using Cufflinks v2.0.2. Differential expression was determined with CuffDiff, using $\chi^{2}$ tests with 1 degree of freedom and 2-tailed $P$ values to assess statistical significance (50). Adjusted $P$ values were calculated using a Bonferonni correction for multiple testing.

Fisher's exact tests for comparing proportions were performed using GraphPad Prism v.7 (GraphPad Software Inc.). A 2-tailed $P$ value less than 0.05 was considered significant. 


\section{Author contributions}

$\mathrm{KME}$ and MME contributed equally to this manuscript. KME, MME, and RD conceived the project, designed and carried out the experiments, and analyzed the results. TW, ABK, EM, and MRD carried out specific steps of experiments. HL, FL, AH, BL, and JQ provided expertise in data analysis and statistical analysis. MLF and MB provided guidance on study design and manuscript writing. KME and RD were primarily responsible for the composition of the manuscript, and $\mathrm{RD}$ was the overall principal investigator for the project.

\section{Acknowledgments}

The authors gratefully acknowledge the advice of Simon Gayther, Kate Lawrenson, Paul Pharoah, and Siddartha Kar in the preparation of this work. This work was supported by funding from the Ruth N. White Research Fellowship in Gynecologic Oncology (KME, MRD), the Sperling Family Fund Fellowship (KME, MRD), the Deborah and Robert First Fund (KME, MRD, RD), the Ruth L. Kirschstein National Research Service Award F31-CA176995-01A1 (MME), the National Cancer Institute at the NIH P50-CA083636 (RD), NIH U01-CA152990 (RD), NIH R21-CA156021 (RD), the Dr. Miriam and Sheldon G. Adelson Medical Research Foundation (RD), the Honorable Tina Brozman Foundation (RD), the Penn Presbyterian George L. and Emily McMichael Harrison Fund for Research in Obstetrics and Gynecology (AB-K), and NIH R01CA193910 (MLF).

Address correspondence to: Ronny Drapkin, Penn Ovarian Cancer Research Center, Department of Obstetrics and Gynecology, Perelman School of Medicine, University of Pennsylvania, Biomedical Research Building II/III, Room 1215, 421 Curie Boulevard, Philadelphia, Pennsylvania 19104, USA. Phone: 215.746.3973; E-mail: rdrapkin@mail.med.upenn.edu.

1. Carroll JS, et al. Chromosome-wide mapping of estrogen receptor binding reveals long-range regulation requiring the forkhead protein FoxA1. Cell. 2005;122(1):33-43.

2. Shchuka VM, et al. Chromatin Dynamics in Lineage Commitment and Cellular Reprogramming. Genes (Basel). 2015;6(3):641-661.

3. Li J, et al. YY1 regulates melanocyte development and function by cooperating with MITF. PLoS Genet. 2012;8(5):e1002688

4. Laurette P, et al. Transcription factor MITF and remodeller BRG1 define chromatin organisation at regulatory elements in melanoma cells. Elife. 2015;4.

5. Chi P, et al. ETV1 is a lineage survival factor that cooperates with KIT in gastrointestinal stromal tumours. Nature. 2010;467(7317):849-853.

6. McGill GG, et al. Bcl2 regulation by the melanocyte master regulator Mitf modulates lineage survival and melanoma cell viability. Cell. 2002;109(6):707-718.

7. West JA, et al. A role for Lin28 in primordial germ-cell development and germ-cell malignancy. Nature. 2009;460(7257):909-913.

8. Laury AR, et al. A comprehensive analysis of PAX8 expression in human epithelial tumors. Am J Surg Pathol. 2011;35(6):816-826.

9. Laury AR, et al. PAX8 reliably distinguishes ovarian serous tumors from malignant mesothelioma. Am J Surg Pathol. 2010;34(5):627-635.

10. Mittag J, Winterhager E, Bauer K, Grümmer R. Congenital hypothyroid female pax8-deficient mice are infertile despite thyroid hormone replacement therapy. Endocrinology. 2007;148(2):719-725.

11. Tone AA, et al. The role of the fallopian tube in ovarian cancer. Clin Adv Hematol Oncol. 2012;10(5):296-306.

12. Roh MH, Kindelberger D, Crum CP. Serous tubal intraepithelial carcinoma and the dominant ovarian mass: clues to serous tumor origin?. Am J Surg Pathol. 2009;33(3):376-383.

13. Perets R, Drapkin R. It's Totally Tubular....Riding The New Wave of Ovarian Cancer Research. Cancer Res. 2016;76(1):10-17.

14. Jones PM, Drapkin R. Modeling High-Grade Serous Carcinoma: How Converging Insights into Pathogenesis and Genetics are Driving Better Experimental Platforms. Front Oncol. 2013;3:217.

15. Karst AM, Levanon K, Drapkin R. Modeling high-grade serous ovarian carcinogenesis from the fallopian tube. Proc Natl Acad Sci U S A. 2011;108(18):7547-7552.

16. Levanon $\mathrm{K}$, et al. Primary ex vivo cultures of human fallopian tube epithelium as a model for serous ovarian carcinogenesis. Oncogene. 2010;29(8):1103-1113.

17. Perets R, et al. Transformation of the fallopian tube secretory epithelium leads to high-grade serous ovarian cancer in Brca; Tp53;Pten models. Cancer Cell. 2013;24(6):751-765.

18. Elias KM, et al. Beyond genomics: critical evaluation of cell line utility for ovarian cancer research. Gynecol Oncol. 2015;139(1):97-103.

19. Domcke S, Sinha R, Levine DA, Sander C, Schultz N. Evaluating cell lines as tumour models by comparison of genomic profiles. Nat Commun. 2013;4:2126.

20. Anglesio MS, et al. Type-specific cell line models for type-specific ovarian cancer research. PLoS One. 2013;8(9):e72162.

21. Mitra AK, et al. In vivo tumor growth of high-grade serous ovarian cancer cell lines. Gynecol Oncol. 2015;138(2):372-377.

22. Hua G, et al. YAP induces high-grade serous carcinoma in fallopian tube secretory epithelial cells. Oncogene. 2016;35(17):2247-2265 
23. Karst AM, Drapkin R. Primary culture and immortalization of human fallopian tube secretory epithelial cells. Nat Protoc. 2012;7(9):1755-1764.

24. Cheung HW, et al. Systematic investigation of genetic vulnerabilities across cancer cell lines reveals lineage-specific dependencies in ovarian cancer. Proc Natl Acad Sci U S A. 2011;108(30):12372-12377.

25. Liu T, et al. Cistrome: an integrative platform for transcriptional regulation studies. Genome Biol. 2011;12(8):R83.

26. De Leo R, Miccadei S, Zammarchi E, Civitareale D. Role for p300 in Pax 8 induction of thyroperoxidase gene expression. J Biol Chem. 2000;275(44):34100-34105.

27. Bopp D, Burri M, Baumgartner S, Frigerio G, Noll M. Conservation of a large protein domain in the segmentation gene paired and in functionally related genes of Drosophila. Cell. 1986;47(6):1033-1040.

28. Frigerio G, Burri M, Bopp D, Baumgartner S, Noll M. Structure of the segmentation gene paired and the Drosophila PRD gene set as part of a gene network. Cell. 1986;47(5):735-746.

29. Mansouri A, Goudreau G, Gruss P. Pax genes and their role in organogenesis. Cancer Res. 1999;59(7 Suppl):1707s-1709s; discussion $1709 \mathrm{~s}$.

30. Chalepakis G, Fritsch R, Fickenscher H, Deutsch U, Goulding M, Gruss P. The molecular basis of the undulated/Pax-1 mutation. Cell. 1991;66(5):873-884.

31. Czerny T, Schaffner G, Busslinger M. DNA sequence recognition by Pax proteins: bipartite structure of the paired domain and its binding site. Genes Dev. 1993;7(10):2048-2061.

32. Mansouri A, Hallonet M, Gruss P. Pax genes and their roles in cell differentiation and development. Curr Opin Cell Biol. 1996;8(6):851-857.

33. Di Palma T, Lucci V, de Cristofaro T, Filippone MG, Zannini M. A role for PAX8 in the tumorigenic phenotype of ovarian cancer cells. BMC Cancer. 2014;14:292.

34. Ruiz-Llorente S, et al. Genome-wide analysis of Pax8 binding provides new insights into thyroid functions. BMC Genomics. 2012;13:147.

35. Clauss A, et al. Overexpression of elafin in ovarian carcinoma is driven by genomic gains and activation of the nuclear factor kappaB pathway and is associated with poor overall survival. Neoplasia. 2010;12(2):161-172.

36. Labidi-Galy SI, et al. Elafin drives poor outcome in high-grade serous ovarian cancers and basal-like breast tumors. Oncogene. 2015;34(3):373-383.

37. Drapkin R, et al. Human epididymis protein 4 (HE4) is a secreted glycoprotein that is overexpressed by serous and endometrioid ovarian carcinomas. Cancer Res. 2005;65(6):2162-2169.

38. Flesken-Nikitin A, Hwang CI, Cheng CY, Michurina TV, Enikolopov G, Nikitin AY. Ovarian surface epithelium at the junction area contains a cancer-prone stem cell niche. Nature. 2013;495(7440):241-245.

39. Sun Y, Jia X, Wu X. High Expressions of Lgr5 and ALDH1 in Primary Epithelial Ovarian Cancer Correlate with Advanced Tumor Stage and Grade as well as Poor Prognosis of the Patients [published online ahead of print June 20, 2015]. Gynecol Obstet Invest. doi: 10.1159/000431222.

40. Ren Y, et al. Targeted tumor-penetrating siRNA nanocomplexes for credentialing the ovarian cancer oncogene ID4. Sci Transl Med. 2012;4(147):147ra112.

41. Kountourakis P, et al. Expression and prognostic significance of kallikrein-related peptidase 8 protein levels in advanced ovarian cancer by using automated quantitative analysis. Thromb Haemost. 2009;101(3):541-546.

42. Magklara A, et al. The human KLK8 (neuropsin/ovasin) gene: identification of two novel splice variants and its prognostic value in ovarian cancer. Clin Cancer Res. 2001;7(4):806-811.

43. Zhang $\mathrm{X}$, et al. Identification of focally amplified lineage-specific super-enhancers in human epithelial cancers. Nat Genet. 2016;48(2):176-182.

44. Hansen CG, Moroishi T, Guan KL. YAP and TAZ: a nexus for Hippo signaling and beyond. Trends Cell Biol. 2015;25(9):499-513.

45. Jolma A, et al. DNA-dependent formation of transcription factor pairs alters their binding specificity. Nature. 2015;527(7578):384-388.

46. Zanconato F, et al. Genome-wide association between YAP/TAZ/TEAD and AP-1 at enhancers drives oncogenic growth. Nat Cell Biol. 2015;17(9):1218-1227.

47. Stein C, et al. YAP1 Exerts Its Transcriptional Control via TEAD-Mediated Activation of Enhancers. PLoS Genet. 2015;11(8):e1005465.

48. Cejas P, et al. Chromatin immunoprecipitation from fixed clinical tissues reveals tumor-specific enhancer profiles. Nat Med. 2016;22(6):685-691.

49. Anders S, Huber W. Differential expression analysis for sequence count data. Genome Biol. 2010;11(10):R106.

50. Trapnell C, et al. Differential gene and transcript expression analysis of RNA-seq experiments with TopHat and Cufflinks. Nat Protoc. 2012;7(3):562-578. 